\title{
Caracterización de espumas de pizarra con adiciones de clínquer de cemento
}

\author{
L.E.G. CAMBRONERO, J.M. RUIZ-ROMÁN, J.M. RUIZ-PRIETO \\ Dept. Ingeniería de Materiales, ETSI Minas-UPM, \\ Ríos Rosas 21, \\ 28003 Madrid
}

\begin{abstract}
La descomposición de los componentes de la Pizarra durante su calentamiento en condiciones no oxidantes conduce a la obtención de espumas. Así, mediante la compactación en matriz a $80 \mathrm{MPa}$ de polvos de Pizarra de menos de 80 $\mu \mathrm{m}$ de tamaño de partícula y con adición de un aglomerante, se obtiene una densidad en verde próxima a 1,8 g/ $\mathrm{cm}^{3}$. El calentamiento de estos compactos en atmósfera de $\mathrm{N}_{2}-5 \% \mathrm{H}_{2}$ permite alcanzar densidades de $2,2 \mathrm{~g} / \mathrm{cm}^{3}$ a $1100^{\circ} \mathrm{C}$, mientras que a $1150^{\circ} \mathrm{C}$ la espumación del compacto conduce a densidades inferiores a $0,4 \mathrm{~g} / \mathrm{cm}^{3}$. La adición de Clínquer de cemento Portland $\left(5 \%\right.$ y $10 \%$ en peso) modifica la densidad de la espuma a $1150^{\circ} \mathrm{C}$, teniendo que elevarse la temperatura de sinterización a $1175{ }^{\circ} \mathrm{C}$ para alcanzarse espumas con densidades próximas a $0,4 \mathrm{~g} / \mathrm{cm}^{3} \mathrm{con} \mathrm{un} 10 \% \mathrm{de}$ Clínquer. Estas espumas con Clínquer presentan un elevado tamaño de poro, así como una resistencia a compresión en el intervalo de resistencias de los vidrios espumados: 0,4-1,5MPa.
\end{abstract}

Palabras clave: Pizarras, Clínquer, vidrio espumado.

\section{Characterization of slate foams with portland clinker additions}

The decomposition of constituents of slates under non-oxidizing atmosphere leads to the manufacturing of foams. Thus die pressing at $80 \mathrm{MPa}$ of slates powders (particle size $<80$ microns) mixed with a solid agglomerate, allows a green density close to $1,8 \mathrm{~g} / \mathrm{cm}^{3}$. The heating of compacts under $\mathrm{N}_{2}-5 \% \mathrm{H}_{2}$ atmosphere allows to reach a final density of $2,2 \mathrm{~g} / \mathrm{cm}^{3}$ at $1050^{\circ} \mathrm{C}$, meanwhile at $1150^{\circ} \mathrm{C}$ the compact foaming leads to densities lower than $0,4 \mathrm{~g} / \mathrm{cm}^{3}$. Clínquer addition $(5 \%$ and $10 \%$ ) modifies the foam density and pore size at $1150^{\circ} \mathrm{C}$. It is necessary to increase the sintering temperature up to $1175^{\circ} \mathrm{C}$ to achieve densities close to $0,4 \mathrm{~g} / \mathrm{cm}^{3}$ when a $10 \%$ of Clínquer is added. The foams with Clínquer shows a high pore size and a compression strength within the range of glass foams: $0.4-1.5 \mathrm{MPa}$.

Keywords: Slates, Clinquer, glass foam.

\section{INTRODUCCIÓN}

Las espumas de Pizarra (1) pueden considerarse por una parte como un caso particular de las espumas de vidrio al estar constituidas por una red de vidrio que aglomera diversos minerales, pero también se pueden clasificar como espumas de cerámica vítrea (2) dada la presencia de esta fase no vítrea. La fabricación de espumas de vidrio de poro cerrado, que comienza alrededor de 1930, se divide en dos métodos: a) la mezcla de polvo fino de vidrio y un agente espumante (2-4) que se consolidan y se tratan térmicamente; $\mathrm{y} \mathrm{b})$ el paso de un gas $\left(\mathrm{CO}_{2}\right.$, aire, vapor de agua) en una masa de vidrio fundido $(5,6)$. La espuma de vidrio resultante es un material ligero, rígido, aislante térmico y acústico (7), resistente al agua, a los agentes químicos, las bacterias y no inflamable (8). Cuando se emplea la vía de la mezcla de polvos, seguida de su consolidación y calentamiento del compacto obtenido, las propiedades finales de la espuma van a depender de la viscosidad de la fase líquida (5) que se obtenga, el mecanismo de espumación del agente espumante (descomposición de carbonatos, reacción de oxidación con la atmósfera, etc), el tamaño inicial de las partículas de vidrio y el ciclo de calentamiento. Con le control de estos parámetros se obtienen (8) densidades de $0,13-0,3 \mathrm{~g} / \mathrm{cm}^{3}$ y resistencias a compresión de 0,4 MPa a 6,0 MPa en espumas de vidrio. Cuando se busca una espuma cerámica vítrea (2), primero se obtiene la espumación de la fase vítrea y posteriormente, se desvitrifica para que esta operación no interfiera con la espumación.

Por otra parte la viabilidad de obtener espumas a partir de las Pizarras, permitiría ampliar el campo de aplicaciones de este material y plantear la utilización de residuos de Pizarra como materia prima del sector cerámico, al igual que esta sucediendo con otros residuos industriales (9).

En el presente trabajo se aprovecha el hinchamiento con la temperatura (1) de las Pizarras, para obtener espumas de vidrio y fases minerales con una estructura de poros cerrados, gracias a la formación de una fase vítrea y a las reacciones de descomposición que tiene lugar a alta temperatura que van a genera los gases necesarios para la espumación. Entre estas reacciones se encuentran las oxidaciones del carbono (en forma de grafito), los sulfatos 
o la pirita (3) y la descomposición de grupos hidroxilos todavía presentes en la Pizarra (por descomposición de los filo silicatos y otros minerales), que generan una mezcla de $\mathrm{CO} / \mathrm{CO}_{2}(8,10-11)$ en atmósferas no oxidantes, que permite que se alcance la espumación del vidrio fundido. Así mismo son estudiadas en este trabajo la modificación que provoca la adición de Clínquer de cemento Pórtland (bien Gris o Blanco), un material de elevada estabilidad térmica $(12,13)$ y utilizado por otros autores (4) como adición en la obtención de espumas, en las propiedades y la composición de la espuma final y de su fase vítrea.

\section{PROCEDIMIENTO EXPERIMENTAL}

2.1 Materia prima.

Los polvos de Pizarra estudiados (1) proceden de los lodos de decantación y filtrado de las plantas de transformación de las explotaciones de la zona de Valdeorras (Orense). Los lodos fueron secados en horno a $120^{\circ} \mathrm{C}$ durante $24 \mathrm{~h}$ en atmósfera de aire estática y posteriormente tamizados para obtener polvos con tamaños inferiores a $80 \mu \mathrm{m}$. Los constituyentes de la Pizarra (13) varían con la procedencia pero, de forma general, están formadas por filosilicatos, cuarzo y minerales accesorios (caolinita, calcita, sulfuros de hierro, materia carbonosa,...). El análisis por difracción de rayos $X(D R X)$ sobre polvo de Pizarra $(<45 \mu \mathrm{m})$ indica que los principales constituyentes mineralógicos (1) son: Chamosita, Cuarzo, Moscovita y Feldespato. Respecto a los valores de la composición química de la Pizarra, obtenida por fluorescencia de rayos X, se indican en la Tabla I. Así mismo se recogen en dicha tabla la composición química y de los constituyentes del Clínquer Blanco y Clínquer Gris $(12,13)$ empleados, ambos con un tamaño de partícula $<45 \mu \mathrm{m}$. Así mismo se indican en la Tabla I los contenidos porcentuales de los componentes del Clínquer (14): $\mathrm{Ca}_{3} \mathrm{SiO}_{5}$ (C3S), $\mathrm{Ca}_{2} \mathrm{SiO}_{4}(\mathrm{C} 2 \mathrm{~S}), \mathrm{Ca}_{4} \mathrm{Al}_{2} \mathrm{Fe}_{2} \mathrm{O}_{10}(\mathrm{C} 4 \mathrm{AF})$ y $\mathrm{Ca}_{3} \mathrm{Al}_{2} \mathrm{O}_{6}(\mathrm{C} 3 \mathrm{~A})$.
$5 \%$ y $10 \%$ en peso de Clínquer Gris o de Clínquer Blanco se mezclaron con un aglomerante para su consolidación. Se adicionó en todos los casos un $5 \%$ en peso de cera, dado que la adición de agua como aglomerante empleado por otros autores (9) reaccionaría con el Clínquer, y se conformaron por compactación uniaxial en matriz flotante a $80 \mathrm{MPa}$ en cilindros de $25 \mathrm{~mm}$ de diámetro y unos $17 \mathrm{~mm}$ de altura. En estas condiciones se alcanzan una densidad en verde de 1,77$1,82 \mathrm{~g} / \mathrm{cm}^{3}$. Los compactos se calentaron en atmósfera de $\mathrm{N}_{2}-$ $5 \% \mathrm{H}_{2}$ durante 30 minutos a temperaturas de $1100{ }^{\circ} \mathrm{C}, 1150^{\circ} \mathrm{C}$ y $1175^{\circ} \mathrm{C}$, empleando tubos metálicos redondos y partidos longitudinalmente para facilitar la posterior extracción de la espuma. Para reducir la adhesión de la fase vítrea que se genera durante el calentamiento de los compactos a las paredes del molde metálico, este se recubrió con una película de Clínquer Blanco, por presentar una baja reactividad con el molde en el rango de temperaturas empleado.

Tras el calentamiento, las probetas obtenidas fueron mecanizadas mediante hilo de diamante para obtener muestras de $20 \times 20 \mathrm{~mm}$ de sección para determinar su densidad (aplicando el principio de Arquímedes) y su resistencia a compresión a una velocidad de $5 \mathrm{~mm} / \mathrm{min}$. Así mismo parte de las probetas se molieron y el polvo obtenido y desorientado $(<45 \mu \mathrm{m})$ se analizó mediante DRX para conocer la composición de la espuma.

\section{RESULTADOS Y DISCUSIÓN}

3.1 Comportamiento térmico y composición de las mezclas de Pizarra y Clínquer

Diversos estudios sobre la alteración al aire de la Pizarra con la temperatura $(3,14,15)$ señalan las distintas transformaciones (deshidratación de las fases hidratadas como los filo silicatos, oxidación de los sulfuros de hierro, oxidación del carbono presente, descomposición de los

TABLA 1. COMPOSICIÓN DE LOS MATERIALES EMPLEADOS.

\begin{tabular}{|c|c|c|c|c|c|c|c|c|c|c|}
\hline & $\% \mathrm{SiO}_{2}$ & $\% \mathrm{Al}_{2} \mathrm{O}_{3}$ & $\% \mathrm{Fe}_{2} \mathrm{O}_{3}$ & $\% \mathrm{MgO}$ & $\% \mathrm{CaO}$ & $\% \mathrm{~K}_{2} \mathrm{O}$ & \multicolumn{4}{|c|}{ Otros } \\
\hline Pizarra & 54,68 & 23,52 & 9,95 & 3,75 & 0,54 & 4,71 & \multicolumn{4}{|c|}{$\begin{array}{c}1,25 \mathrm{TiO}_{2^{\prime}} 1,25 \% \mathrm{Na}_{2} \mathrm{O} \\
0,08 \% \mathrm{MnO}^{2}, 26 \% \mathrm{P}_{2} \mathrm{O}_{5}\end{array}$} \\
\hline & \multicolumn{6}{|c|}{ Composición química } & \multicolumn{4}{|c|}{ Componentes del Clínquer } \\
\hline Clínquer & $\% \mathrm{SiO}_{2}$ & $\% \mathrm{Al}_{2} \mathrm{O}_{3}$ & $\% \mathrm{Fe}_{2} \mathrm{O}_{3}$ & $\% \mathrm{MgO}$ & $\% \mathrm{CaO}$ & $\% \mathrm{CaO}$ libre & $\% \mathrm{C} 3 \mathrm{~S}$ & $\% \mathrm{C} 2 \mathrm{~S}$ & $\% \mathrm{C} 3 \mathrm{~A}$ & $\% \mathrm{C} 4 \mathrm{AF}$ \\
\hline Gris & 20.26 & 6.11 & 3.50 & 1.14 & 65.70 & 1.22 & 63,22 & 12,14 & 10,18 & 10,65 \\
\hline Blanco & 23,07 & 4,78 & 0,36 & 0,74 & 68,95 & 0,59 & 70,44 & 13,08 & 11,97 & 1,10 \\
\hline
\end{tabular}

Finalmente, el comportamiento térmico de las mezclas de polvos de Pizarra $+10 \%$ en peso de Clínquer (Blanco o Gris) se estudió mediante análisis termo gravimétrico (TG), análisis térmico diferencial(TDA) y STDA ( Single Differential Thermal Analisis), así como de Pizarra sin adiciones, en corriente de nitrógeno, con vasija de alúmina y a una velocidad de calentamiento de $10{ }^{\circ} \mathrm{C} / \mathrm{min}$ hasta alcanzar los $1275^{\circ} \mathrm{C}$.

\subsection{Fabricación y caracterización de materiales.}

Los polvos de Pizarra y las mezclas de Pizarra con un carbonatos, ...) que se suceden (3) hasta alcanzarse, a partir de $1150^{\circ} \mathrm{C}$, un material con una composición final constituida por espinelas, mullita, $\beta-\mathrm{Fe}_{2} \mathrm{O}_{3}$, hematites, cuarzo, $\gamma$-alúmina y una fase vítrea continua (16).

La evolución térmica de la Pizarra (Curvas TG-ATDSTDA, Figura 1a,) se divide en dos intervalos o etapas de fuerte cambio en peso: hasta $580{ }^{\circ} \mathrm{C}$ (intervalo 1, Figura 1a), y de $580^{\circ} \mathrm{C}$ a $1200{ }^{\circ} \mathrm{C}$ (intervalo 2, Figura 1a), con unas variaciones en peso del $2,4 \%$ y $3.31 \%$, siendo la pérdida total por el calentamiento del $4.73 \%$ (intervalo 3, Figura 1a). Respecto a la Pizarra con adiciones de Clínquer, las variaciones en masa se sitúan en $5.10 \%$ para un $10 \%$ de 

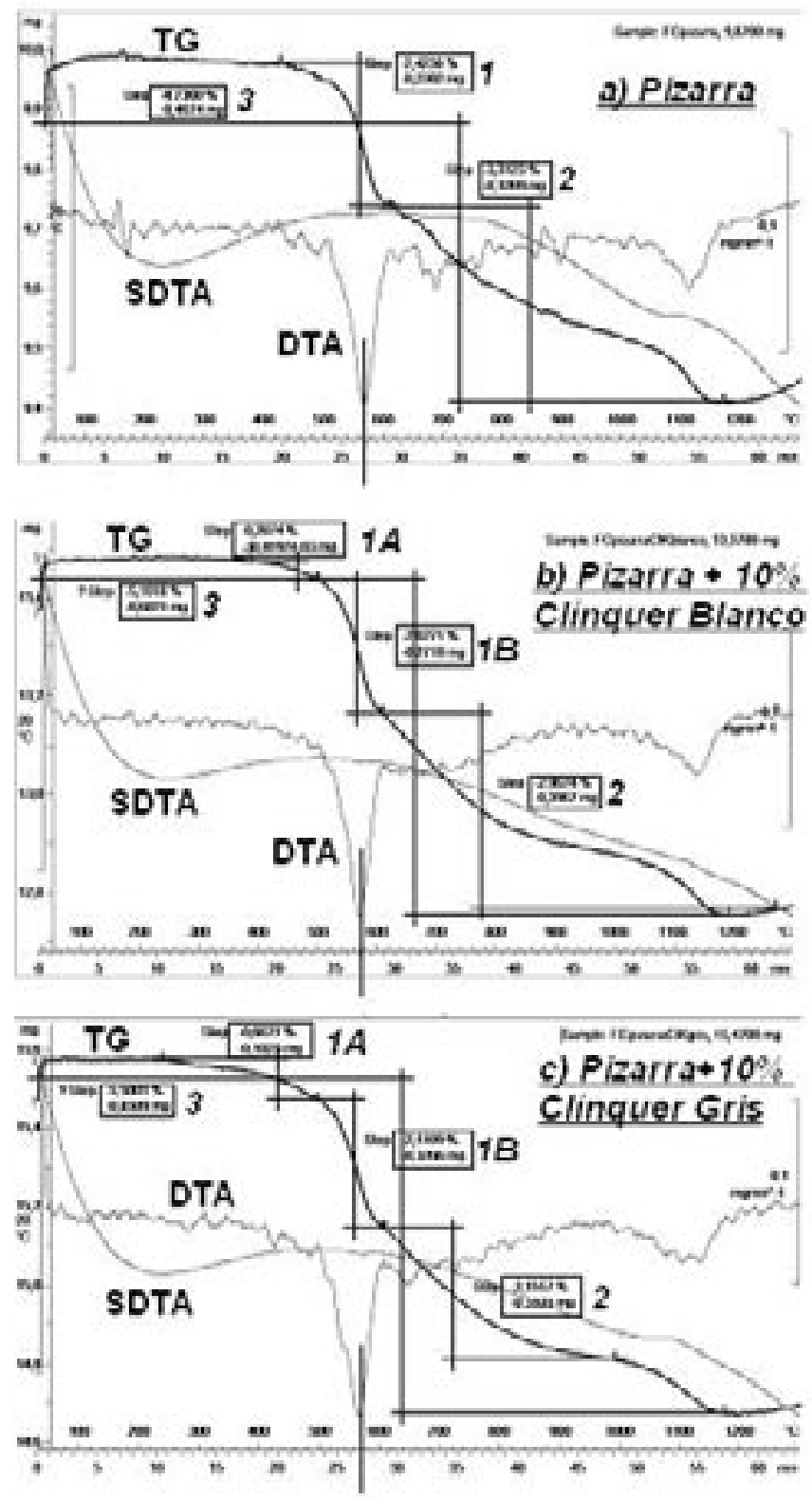

Fig. 1- TG , ATG y SDTA de los polvos de pizarra y de las mezclas Pizarra+ $10 \%$ Clínquer Blanco y Pizarra+ 10\%Clínquer Gris.

Clínquer Blanco (Figura 1b, intervalo 3) y $5.5 \%$ para un $10 \%$ de Clínquer Gris (Figura 1c, intervalo 3). Para ambas mezclas de Pizarra y Clínquer, el intervalo 1 (20-450 $\left.{ }^{\circ} \mathrm{C}\right)$ se desdobla en dos $1 \mathrm{~A}$ y $1 \mathrm{~B}$, sin que se haya determinado las reacciones que tienen lugar a estas bajas temperaturas, pero donde la posible hidratación del Clínquer, que no ha sido secado previamente, podría influir. Estos resultados de los análisis térmicos TG -DTA, y en menor medidas de las curvas STDA por ser muy parecidas en los tres casos, señalan que en todos los materiales, la pérdida de masa se acelera hasta los $560^{\circ} \mathrm{C}$ (reacción exotérmica de la curva DTA, Figura 1a,1b y1c) como consecuencia de la reacción de deshidratación de los filosilicatos . Sin embargo, las reacciones posteriores entre $620^{\circ} \mathrm{C}$ y $1000^{\circ} \mathrm{C}$ debidas a las oxidaciones y descomposiciones de carbonatos presentes en la Pizarra y señaladas por una sucesión de picos exotérmicos (Figura 1a, curva DTA), no sedan en la Pizarra
Intensidad (u.a.)
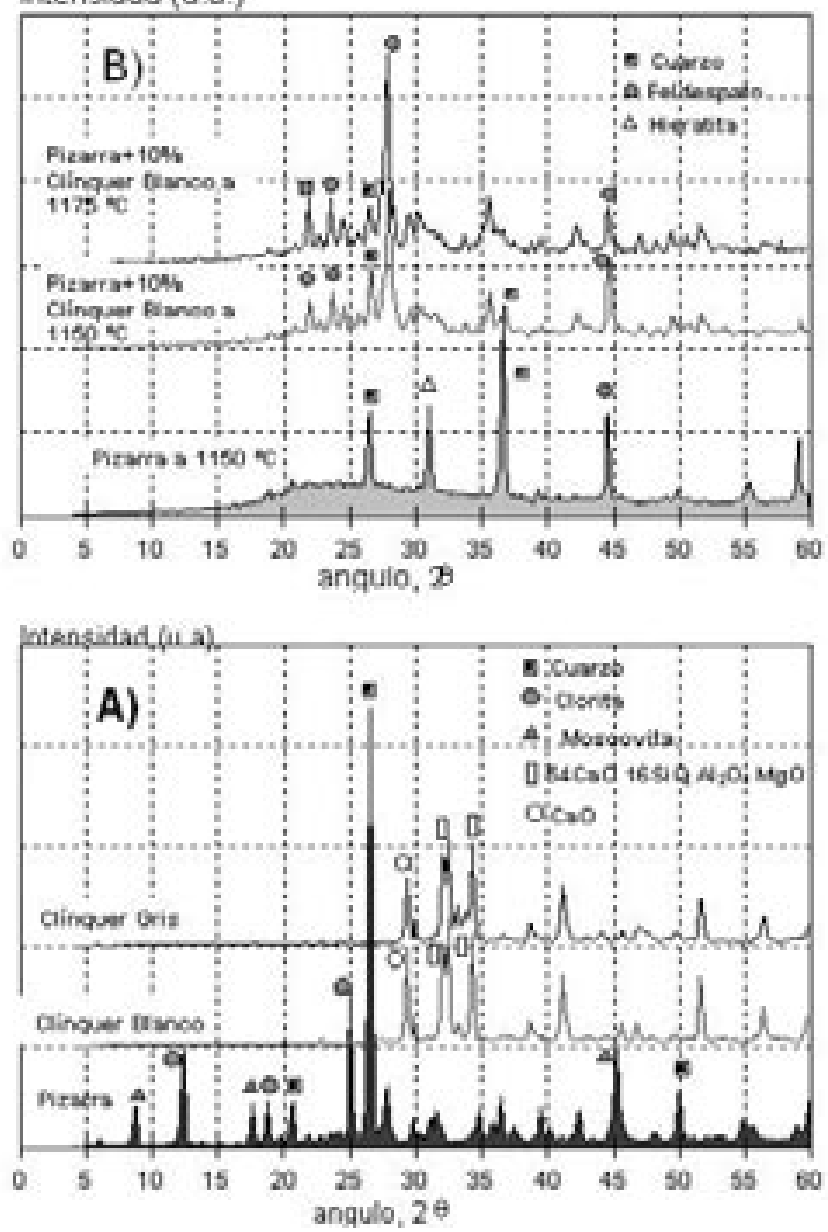

Fig. 2- Difracción de Rayos X de: A) las materias primas y B) de las espumas de Pizarra $\left(\right.$ a $1150^{\circ} \mathrm{C}$ ) y de Pizarra con $10 \%$ Clínquer Blanco $\left(\mathrm{a} 1150^{\circ} \mathrm{C}\right.$ y $1175^{\circ} \mathrm{C}$ ).

con Clínquer, por lo que estas reacciones conducen a mayor pérdida en peso total en la Pizarra (3,31\%), que en el Clínquer Blanco $(2,96 \%)$ o Gris $(2,15 \%)$. De asociarse esta menor perdida en peso con las reacciones de generación de gases, se obtendrá una menor espumación, y con ello una menor densidad, cuando se adicione Clínquer a la Pizarra. Así mismo para temperaturas superiores a $1000^{\circ} \mathrm{C}$ hay en todos los casos un pico exotérmico (curvas TG de la Figura 1) debido a la presencia de la fase liquida. Por la altura del pico cabe indicar una menor cantidad de esta fase en el caso de las adiciones de Clínquer, dada la sustitución de un $10 \%$ de Pizarra por Clínquer (Gris o Blanco).

Después de su calentamiento a temperaturas entre 1150 ${ }^{\circ} \mathrm{C}$ y $1175^{\circ} \mathrm{C}$, las espumas de Pizarra + Clínquer presentaron un color gris oscuro, al igual que las espumas de Pizarra sin adiciones (1). En todos los casos se obtiene una fase vítrea contínua (que aumenta la línea base en los difractogramas respecto de los obtenidos con las materias primas, Figura 2A) que aglomera una serie de minerales (Figura 2B) entre los que se encuentran pequeñas cantidades de cuarzo y feldespato $\left([\mathrm{Ca}, \mathrm{Na}]\left[\mathrm{Si}, \mathrm{Al}_{4} \mathrm{O}_{8}\right.\right.$ o $\left.\mathrm{CaAlSi}_{3} \mathrm{O}_{8}\right)$. Este último procede de la combinación de los componentes de la Pizarra con los componentes del Clínquer (Gris o Blanco), por cuanto que este feldespato no esta presente en las espumas de Pizarra sin adiciones (Figura 2A). En relación con la 
composición final de la espuma de Pizarra + Clínquer, las fases minerales presentes a $1150^{\circ} \mathrm{C}$ obtenidos por difracción (XRD) son las mismas si se emplea Clínquer Gris o Blanco, por lo que se ha omitido su difractograma en la Figura 2B, si bien se ha obtenido una aumento de intensidad en el pico del feldespato cuando se trata de Pizarra con Clínquer Gris. Respecto a la Pizarra, tras su calentamiento a $1150^{\circ} \mathrm{C}$ presenta cuarzo, mullita y espinelas de hierro $(1,13,14)$ además de una fase vítrea por la evolución del sistema $\mathrm{FeO}-$ $\mathrm{Al}_{2} \mathrm{O}_{3}-\mathrm{SiO}_{2}$ (15).

\subsection{Desarrollo y propiedades de la estructura porosa.}

A bajas temperaturas $\left(1000^{\circ} \mathrm{C}\right)$ la densidad alcanzada por la Pizarra es elevada $\left(2,18 \mathrm{~g} / \mathrm{cm}^{3}\right)$, y disminuye con la adición de Clínquer a valores de $1,82 \mathrm{~g} / \mathrm{cm}^{3}$ (con $10 \%$ Clínquer Blanco) y $1,66 \mathrm{~g} / \mathrm{cm}^{3}$ (con $10 \%$ Clínquer Gris) debido a la disminución de la fase liquida presente que conduce a la densificación del material. La espumación (Figura 3) se presenta en la

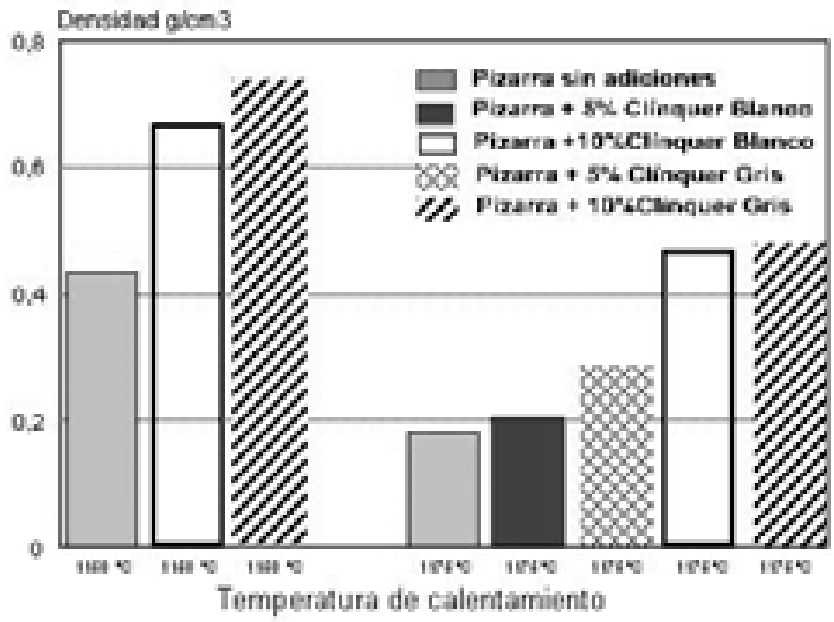

Fig. 3- Densidades de las espumas obtenidas a distintas temperaturas de calentamiento.

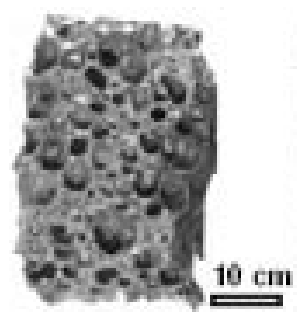

$10 \%-1175 \% \mathrm{C}$

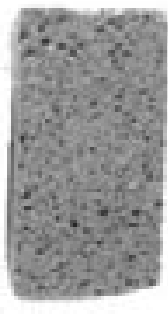

$0 \div-1150^{\circ} \mathrm{C}$

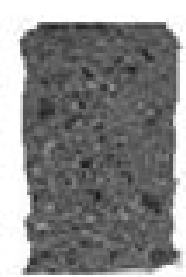

$5 \%-1150 \% \mathrm{C} 10 \%-1150^{\circ} \mathrm{C}$
Fig. 4- Aspecto de las espumas de Pizarra con distintos contenidos de Clínquer Gris a distintas temperaturas de calentamiento.

Pizarra sin adiciones a temperaturas superiores a $\operatorname{los} 1100^{\circ} \mathrm{C}$ (1) debido a la presencia de una fase vitrea fundida y la formación de gases de descomposición o reacción de los constituyentes, junto la perdida de solubilidad de gases en la fase vítrea . Es necesario alcanzar temperaturas de 1150 ${ }^{\circ} \mathrm{C}$ para que se desarrolle una estructura de poros con un tamaño de poro inferior a $2 \mathrm{~mm}$ (Figura 4) y se obtenga una espuma de vidrio. A esta temperatura $\left(1150^{\circ} \mathrm{C}\right)$ las adiciones de Clínquer reducen la espumación de la Pizarra, en mayor medida conforme se aumenta el contenido del Clínquer (del 5\% al 10\%, Figura 4), y aumentan la densidad final a valores superiores a los $0,4 \mathrm{~g} / \mathrm{cm}^{3}$ (Figura 3) alcanzados en la espuma de Pizarra sin adiciones. El aumento de la temperatura de calentamiento de $1150^{\circ} \mathrm{C}$ a $1175^{\circ} \mathrm{C}$, permite una mayor espumación en todos los materiales, si bien la adición del $5 \%$ o del $10 \%$ de Clínquer hace que a esta mayor temperatura $\left(1175^{\circ} \mathrm{C}\right)$, la densidad sea superior a la obtenida con la Pizarra sin estas adiciones $\left(0,2 \mathrm{~g} / \mathrm{cm}^{3}\right.$, Figura 3$)$. La porosidad alcanza también un tamaño mayor a estas altas temperaturas y es mas heterogénea, alternándose grandes y pequeños poros (Figura 4). Una menor viscosidad de la fase vítrea a esta mayor temperatura en todos los materiales, seria la causa principal de este comportamiento. Además, en la Pizarra con adiciones de Clínquer (tanto si es Blanco o Gris) apenas varía la composición al aumentar la temperatura de $1150^{\circ} \mathrm{C}$ a $1175^{\circ} \mathrm{C}$ a (Figura 2B), disminuyendo ligeramente el contenido en cuarzo y aumentando el del feldespato (Figura 2B), sin modificar la altura de la línea base del difractograma, por lo que no parece que dicho aumento de temperatura se traduzca en un mayor contenido en fase vítrea y con ello estas adiciones van a reducir esta fase. La estabilidad de los feldespatos que se forman en la Pizarra con adiciones de Clínker, reducirían la viscosidad de la fase vítrea fundida al actuar como espesantes.

Finalmente la resistencia a compresión de las espumas se sitúa en el intervalo característico de las espumas de vidrio (8) con valores medios de 1,3MPa para la espuma de Pizarra a $1150^{\circ} \mathrm{C}$ y de $1,5 \mathrm{MPa}$ para las espumas con $10 \%$ de Clínquer, tanto si es Blanco como Gris, a $1175^{\circ} \mathrm{C}$,

\section{CONCLUSIONES}

La adición de Clínquer a los polvos Pizarra procedente de lodos de decantación modifica el comportamiento con la temperatura de los compactos de Pizarra en atmósfera de $\mathrm{N}_{2}-5 \% \mathrm{H}_{2}$. La fase líquida (vítrea) que se genera no presenta las mismas características de viscosidad ni volumen en la mezcla de Pizarra y Clínquer, que en la Pizarra. Por ello es necesario aumentar la temperatura hasta los $1175^{\circ} \mathrm{C}$ para que se desarrolle la estructura porosa de la espuma. No hay importantes diferencias en la propiedades finales de las espumas según se adicione Clínquer Gris o Blanco, obteniéndose en ambos casos a $1175^{\circ} \mathrm{C}$ una estructura con grandes poros, con densidades próximas a $0,3 \mathrm{~g} / \mathrm{cm}^{3}$.

\section{AGRADECIMIENTOS}

Deseamos agradecer a la Comisión Europea por su proyecto CRAFT BRST-CT97.5126 del programa BRITE III, a la CICYT por su Acción Especial MAT 0067456, a Portland Valderrivas S.A. y Cemex S.A por el Clínquer suministrado y a La EPS de Linares (Univ. Jaén) por los ensayos de ATGATD-SDTA. 


\section{REFERENCIAS}

1. L.E.G. Cambronero, J.M. Ruiz Román, J.M. Ruiz Prieto, Obtención de espumas a partir de residuos de Pizarra, Bol. Soc. Esp. Ceram. V., 44, [6], 368-372 (2005)

2. P. Colombo, G. Brusatin, E. Bernardo, G. Scarinci, Inertization and reuse of waste materials by vitrification and fabrication of glass-based products, Solid Stare \& Material Science, 7, 225-239 (2003).

3. A. Casal, J. Grande, Algunas materias primas utilizaveis no fabrico de agregados leves. Congreso de Ingeniería, 22-29 Noviembre, Oporto (1978).

4. G. vargas, f.Vázquez, J. López, J. Méndez, M. Méndez, P. Pena, Espumado de mezclas de silicato de sodio-wollastonita por microondas, Bol. Soc. Esp. Ceram. V., 43 [1] 71-74 (2004).

5. A.G. Fedorov, L. Pilon, Glass foams: formation, transport properties, and heat, mass, and radiation transfer, J. Non-Crystalline Solids, 311, 154-173 (2002).

6. E. R. Powell, US Patent, 3, 133,820, (1964).

7. U. Fisher, J. Hubalkova, J. Ulbricht, C.G. Aneziris, Foam glass processing for fiber-free automotive silencers, EUROMAT, 5-8 Septiembre, Praga (2005).

8. M. Scheffler, P.Colombo, Cellular Ceramics, Ed. Wiley VCH, 158-174 (2004).

9. M. Aineto, A. Acosta, Las escorias de la central térmica GICC ELCOGAS como materia prima para la síntesis de materiales vitrocerámicos. Parte 1 : Comportamiento en fusión de las escorias GICC y obtención del vidrio original, Bol. Soc. Esp. Ceram. V., 44 [6] 399-404 (2005).
10. P. Faenden, H.G. Schott, Guide to glass, Ed. Chapman \& Hall, London, 186-187 (1992).

11. W.D. Ford (Pittsburg Corning, USA), US Patent, 2, 401, 582 (1946).

12. J.M. Torralba, L.E.G. Cambronero y J.M. Ruiz-Prieto, Viability of using Clínquer Portland as raw material in structural ceramics, J. Am. Ceram. Soc. 74 [11], 90-91 (1995).

13. N. Antón, L.E.G. Cambronero, J.M. Ruiz-Prieto, F. Velasco, J.M. Torralba, Ceramic and Ceramic Matrix composites Based on Clínquer Portland: Sinterability, Key Eng. Mat., 127-131, 407-414 (1997).

14. A. G. De La Torre, M.A.G. Aranda, A. H. De Aza, P. Pena, S. De

AZA, Belite Portland Clínquers. Synthesis and Mineralogical Analysis, Bol. Soc. Esp. Ceram. V., 44 [3] 185-191 (2005).

15. M.A. Rodríguez, F. Rubio, J. Rubio, M.J. Liso, J.L. Oteo, Caracterización estructural y comportamiento térmico de una muestra de Pizarra empleada como material para la edificación, Bol Geol. y Minero, 1, 437445 (1995).

16. A.Casal, J. Grande, Contribucao para o estudio da expansibilidades del xisto ardosifero da region de Valongo, Estudios do SFM, 23, 243-260 (1977).

17. MTVieira, L Catarino, M Oliveira, J Sousa, JM Torralba, LEG Cambronero, FL González-Mesones, A. Victoria, Optimisation of the sintering process of raw material wastes, J. Mat. Proc. Tech., 92-93, 97-161, (1999).

18. J.F. Schairer , K.Yagi, The system $\mathrm{FeO}-\mathrm{Al}_{2} \mathrm{O}_{3}-\mathrm{SiO}_{2}$, Am. J. Sci, 25, 151-160 (1952).

Recibido: 29.11 .05

Aceptado: 23.08 .06 